\title{
COMPOSITE MODELING TO ENHANCE HYDRAULIC STRUCTURES STUDIES
}

\section{Modélisation combinée pour améliorer l'étude des structures hydrauliques}

\author{
Sébastien Erpicum ${ }^{1}$, Benjamin J. Dewals, Pierre Archambeau, Michel Pirotton \\ HECE - ArGEnCo - University of Liege, Chemin des Chevreuils, 1 B52/3, B-4000 Liege, Belgium \\ S.Erpicum@ulg.ac.be, hece@ulg.ac.be
}

\section{KEY WORDS}

Numerical models, physical models, scale models, scale factor, parametric study

\begin{abstract}
Physical modeling and numerical modeling are two efficient analysis approaches in hydraulic engineering. The interactive application of both methods is obviously the more effective response to most flow problems analyses. Indeed, it enables combining the inherent advantages of both approaches, which are complementary, while being beneficial to the delays as well as the quality of the analysis. The paper presents the way composite modeling is applied for years at the HECE - Laboratory of Engineering Hydraulics (University of Liege) to enhance hydraulic structures studies. Besides numerical model validation for which experimental benchmarks constitute the first reliable data source, simultaneous application of both modeling approaches may be envisaged in three different ways. Composite modeling may be used to increase the scale factor of physical models by reducing the layout of the real structure to be modeled, to provide a better answer to specific problems than a single approach study or to maximize the efficiency of experimental tests by reducing the range of variation of the unknown parameters to be tested. For each of these three issues depicted in the paper, several examples show how the combined use of efficient numerical solvers together with physical scale models enables to increase the overall quality and scope of the analyses while decreasing the delays and possibly the costs.
\end{abstract}

\section{INTRODUCTION}

Physical modeling has always been widely used in hydraulic engineering for research as well as project design. It consists in building, with a degree of complexity depending on the goals of the application, a model of the hydraulic systems to be studied [1]. This type of model enables to reproduce, in a controlled environment making a qualitative as well as quantitative analysis possible, the whole complexity of the flows occurring in the studied systems. The main advantage of the physical models lies in their intrinsic capacity to reproduce, provided that adapted scale factor and similarity laws are applied, the complete flow features, even very complex. Moreover, they easily enable physical interactions on the flow patterns and are useful for promoting and communicating on a project. Indeed, they irrefutably demonstrate to the specialist engineer but also to each individual how a structure or a procedure will work [1].

The general use of numerical modeling in hydraulic engineering dates from about twenty five years. It has been developed following the setup of increasingly representative mathematical models as well as more and more robust and accurate resolution schemes, coupled to a tremendous increase and popularization of the numerical computing potentialities. Just like the efficiency and the pertinence of a physical model depend on a judicious choice of the scale factor and the similarity laws, carrying out representative and reliable flow numerical modeling depends on a number of parameters, sometimes still little recognized such as the choice of the mathematical model, of the resolution scheme, of their parameters, the quality and the adequacy of the input data, and in particular the boundary conditions, or the modeler experience. The main advantages of the numerical models are their low application costs compared to those of a scale model building, especially for

\footnotetext{
${ }^{1}$ Corresponding author
} 
large study areas, their flexibility, in terms of geometry for example, or also the ease for tracking the evolution of the unknowns everywhere in the studied system.

Today, the combined application of physical and numerical modeling, called composite modeling, is obviously the more effective response to most flow problems analyses. Indeed, it enables combining the inherent advantages of both approaches, which are complementary, while being beneficial to the delays as well as the quality of the analysis.

This paper presents the way composite modeling is applied for years at the HECE - Laboratory of Engineering Hydraulics (University of Liege) to enhance hydraulic structures studies. Besides numerical model validation for which experimental benchmarks constitute the first reliable data source, simultaneous application of both modeling approaches is envisaged in three different ways. Composite modeling si used to increase the scale factor of physical models by reducing the layout of the real structure to modeled (section 2), to provide a better answer to specific problems than a single approach study (section 3) or to maximize the efficiency of experimental tests by reducing the range of variation of the unknown parameters to be tested (section 4).

For each of these three issues, several examples show how the combined use of efficient numerical solvers together with physical scale models enables to increase the overall quality and scope of the analyses while decreasing the delays and possibly the costs.

\section{INCREASING THE SCALE FACTOR OF PHYSICAL MODELS}

The ability of the numerical models to consider large investigation areas without major constraints is generally used to perform large scale hydraulic analysis of the projects. In addition, such tools enable to precisely define the flow conditions close to a specific area or structure for which a scale model study is requested, for instance because of the complexity of the flow processes or of the structure (energy dissipation, turbulence problems, weir design...). The detailed knowledge of the flow conditions near this specific location authorizes to reduce the layout of the scale model while setting up adequate boundary conditions to keep the physical model representativeness. At constant area in the laboratory, the scale factor of the model may thus be increased, maximizing the accuracy of the physical experimentations while reducing the scale effects.

For instance, such an approach has been successfully applied in the scope of the Raviege dam [5] and the Taoussa project [7] studies, depicted below.

\subsection{Raviege dam}

The Raviege dam, operated by Electricité de France (EDF), is located in South West of France, near the town of Toulouse, on the Agout River. This concrete buttress 40-m high dam was built in 1957 for electricity production. The hydropower plant is located at the dam toe, on the right side of the river. Hydrology calculations of the dam catchment area have been recently updated and showed a new extreme flood far more significant than the one considered at the dam design stage. The dam release capacity is thus not sufficient regarding extreme floods and a project aiming at increasing the dam safety has been studied. Several potential solutions combine the use of the reservoir storage capacity and a new free overflow spillway on top of the dam, beside the existing gates, to face safely the new extreme floods.

Because of the critical issues related to energy dissipation downstream of the dam, it has been decided to study the different rehabilitation solutions by means of a physical model. However, the Raviege dam is located in a bend of the Agout River. Without preliminary analysis of the flow conditions in the reservoir, the scale model layout has to be very large to guarantee the representativeness of the currents close to the dam.

In order to reduce as much as possible the scale model layout in the reservoir and thus to maximize the scale factor at constant area in the laboratory, a numerical study has been performed. A 2D approach, considering turbulence effects by means of a depth averaged k- $\varepsilon$ mathematical model, has been applied. Two computations have been carried out: one to define the full reservoir flow patterns and the other one to define 
the scale model flow patterns. The comparison of the results enables to optimize the scale model dimensions and to define the position and geometry of the discharge repartition wall upstream of the model, while preserving the equivalence between approach currents to the spillways.

A scale model factor of 1:35 has been made possible by this composite approach, leading to a physical model area of $11 \times 6.6 \mathrm{~m}^{2}$ (Fig. 1).

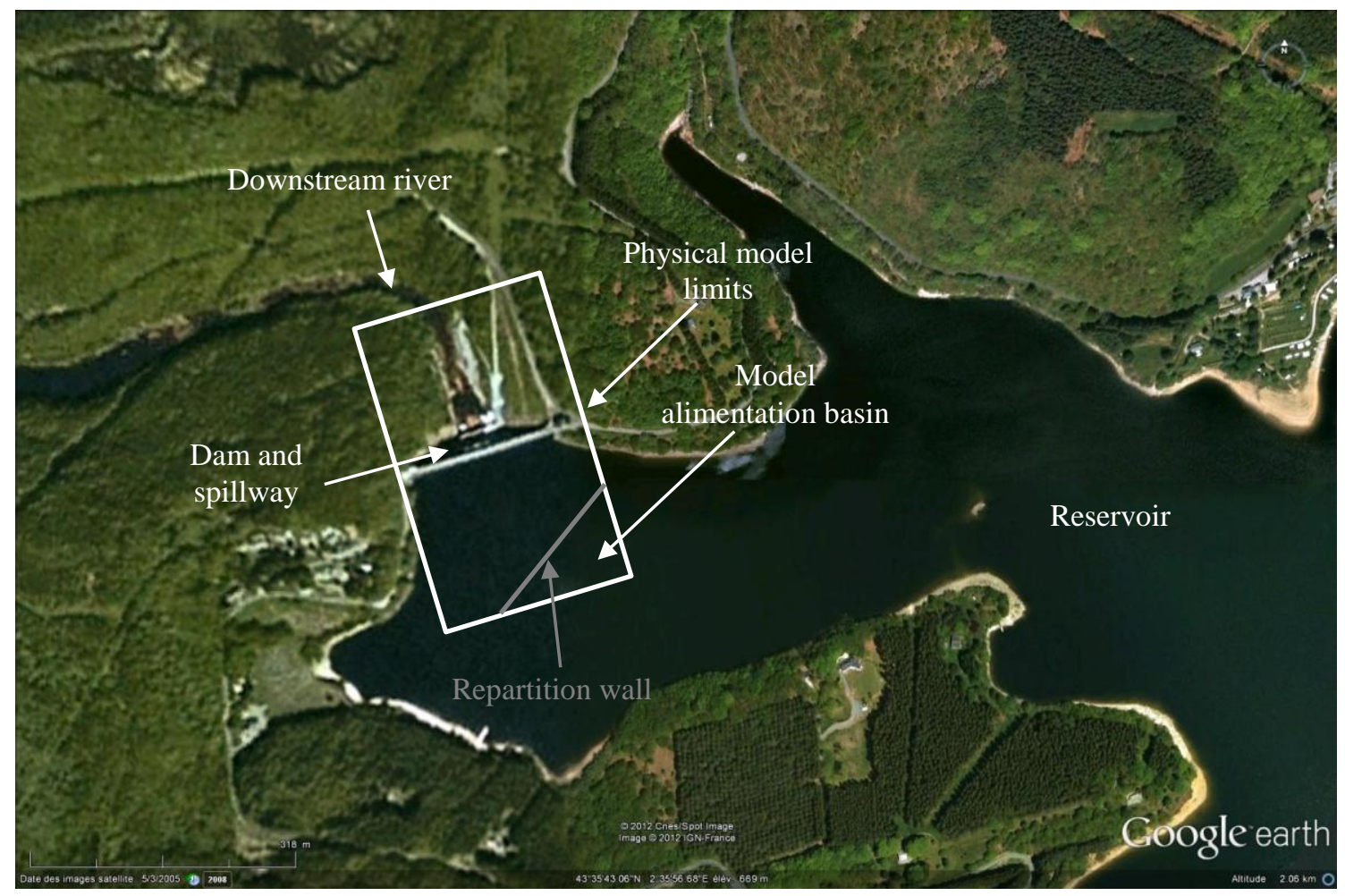

Figure 1: Aerial view of the Raviege dam and 1:35 scale model layout.

\subsection{The Taousa project}

The Taoussa hydraulic project includes a main embankment dam around $15 \mathrm{~m}$ high and 1,300 $\mathrm{m}$ long across the valley of the Niger River, $130 \mathrm{~km}$ upstream of the town of Gao in the eastern part of Mali. This structure has to enable a long-term control of the river discharges to allow the development of farming and economic activities.

The dam is equipped with a hydroelectric power plant counting for 5 groups with an equipment discharge of $75 \mathrm{~m}^{3} / \mathrm{s}$ each, a $12 \mathrm{~m}$ wide lock and a spillway with a release capacity of $3,100 \mathrm{~m} / \mathrm{s}$. The spillway is made of a Creager weir at level $252.75 \mathrm{~m}$ divided in 10 gated notches $8.5 \mathrm{~m}$ wide, separated by $3.3 \mathrm{~m}$ wide piers. A stilling basin $55 \mathrm{~m}$ long is located downstream of the weir. Its bottom elevation is at level $244 \mathrm{~m}$ with a 2-m high step at its downstream extremity. The stilling basin is divided longitudinally in 5 identical parts by walls at level $255 \mathrm{~m}$, extending the weir piers. The spillway has to control the reservoir level in between levels 254.50 and $258.75 \mathrm{~m}$ in annual usual operation and normal conditions. During high flood events, (i.e. discharge higher than 2,500 $\mathrm{m}^{3} / \mathrm{s} / 100$-year flood), the reservoir level could rise to a higher level, in particular up to level $260.80 \mathrm{~m}$ for the 1000 -year flood of $3,100 \mathrm{~m}^{3} / \mathrm{s}$ with 8 gates opened (2 gates blocked or closed for maintenance).

The hydraulic studies of the project have been carried out successively by two complementary approaches: a first step realized on the basis of depth averaged numerical modeling to study the flows at the scale of the reservoir and the river, and a second one using a physical scale model, to detail the spillway operation conditions. Indeed, this last one is a structure with complex hydrodynamics, whose importance to guarantee the satisfactory working of the whole project is crucial. It has thus to be studied carefully using a physical model with the largest scale factor possible. In this scope, the layout of the physical scale model and the location of the water alimentation wall in the model upstream basin have been carefully tuned on the 
basis of an analysis of the numerical results of the reservoir flow modeling in order to increase as much as possible the scale factor while limiting the model area and thus the costs.

The quality of the reduced layout of the physical model has been defined by comparison of the flow fields computed on the basis of either the physical model layout or the whole reservoir. Such an approach provides objective validation of the representativeness of the scale model regarding for instance the spillway alimentation conditions and enables an a posteriori verification by measuring and comparing the flow fields directly in the scale model.

A scale factor of 1:40 has been made possible, leading to a $10 \times 8 \mathrm{~m}^{2}$ physical model with a $2 \times 3 \mathrm{~m}^{2}$ area for the spillway and an upstream reservoir only $3 \mathrm{~m}$ long. Moreover, the application of a combined numerical - experimental approach enables to study the whole of the hydraulic features of the project in less than 6 months.

\section{MOST EFFICIENT ANSWER TO SPECIFIC PROBLEMS}

Physical modeling and numerical modeling are complementary. Physical models enable to reproduce, for all that adapted scale factor and similarity laws are applied, the complete flow features, even very complex. However, they need place and time to be built and are less accurate with decreasing scale factor, and thus with increasing area to model at constant space in the laboratory.

On the other hand, numerical models are particularly suited for large area studies. Indeed, for such applications, they need less time and present lower application costs than physical models, they are more flexible and they don't suffer scale effects. However, for complex or particular flow analysis, they still often have lacks in representativeness as they are directly dependent of the mathematical model used to idealize the physical processes occurring in real flow, or their computation costs are prohibitive for practical case studies when fully representative mathematical models are solved.

It is thus obvious than composite modeling, where each model type is applied for what it is the most suited, provides a better answer to specific problems than a single approach study. This is illustrated in the following paragraphs trough three examples. The first one is related to the Raviege dam study already mentioned in section 2.2, the second one describes the study of a locks complex on the Meuse River in Belgium [6] and the last one is related to the design of a water intake on a river bank.

\subsection{Downstream flows on the Raviege dam project}

Flow conditions in the natural river downstream of the Raviege dam was a critical point of the scale model study depicted in section 2.1. No rating curve is available to define the water level close to the Raviege dam in the range of discharges considered to design the new spillway. However, regarding energy dissipation at the dam toe, the downstream water level is a parameter of pirme importance. This water level and the back water curve in the river reach are directly controlled by the Ponviel dam, $2 \mathrm{~km}$ downstream of the Raviege dam.

To fix this problem, two solutions may be envisaged. The physical model could be extended down to the Ponviel dam. From a practical point of view, this solution is not efficient. Indeed, in that case, either the scale model layout will be very important, or the scale factor will have to be significantly reduced. The second solution, which has been applied in the project, consists in using first a numerical model to compute the rating curve at the physical model extremity, and to use the physical model to focus on energy the dissipation problem at the dam toe.

To compute the rating curve of the natural river, the 2-km long river each has been modeled in one dimension. The reach slope is weak, and the Ponviel dam spillway controls the water depth upstream. The discharge in the river reach is the one spilled at the Raviege dam. Modeling of the back water curves along the whole reach for various discharges and considering different values of the roughness coefficients provides a reliable prediction of the most probable water levels at the downstream extremity of the scale model. Besides defining the downstream boundary condition to be verified on the scale model, these free 
surface elevation data have been used to define the maximum level of the topography to be reproduced in the scale model.

\subsection{Hydraulic study of the $4^{\text {th }}$ Lanaye lock}

The Lanaye lock complex is located in Belgium, $20 \mathrm{~km}$ north from the town of Liege. It links the Albert canal to the waterways network of the Netherlands via the Meuse River. This important European navigation node is equipped with 3 locks ( 2 of $55 \times 7.5 \mathrm{~m}$ and 1 of $136 \times 16 \mathrm{~m}$ ) and a fourth one $(225 \times 25 \mathrm{~m}$ ) is currently under construction. A pumping station and a hydropower plant will also been built in order to cancel the new lock water consumption during droughts and to exploit the $13.68 \mathrm{~m}$ high chute during high flow periods.

The hydraulic study of the new lock and power station project required tremendous modeling works, due to the huge variation of the spatial scales as well as of the complexity of the flows depending on the problem to be solved. The scope of the analysis ranges from the hydrodynamic effect of the lock operation in the downstream and upstream reaches to the design of the power station intake and the evaluation of the energy dissipation in the lock emptying system. In this context, the application of only numerical modeling or only physical modeling is certainly not the most efficient approach. Following a careful preliminary analysis of the problems to deal with, numerical and physical models have been used together, each approach being applied where it was the most suited, pertinent boundary conditions being eventually reported from a model to another.

Coupled 1D-2D numerical modeling [6] has been applied to investigate hydraulic features at the scale of the upstream and downstream reaches, depending on varied locks operation configurations. These simulations also provided the hydraulic data necessary for navigation conditions modeling. At a more focused level, specific numerical models have been used to design the power plant water intake and outlet. On the other hand a physical model has been built to study the energy dissipation in the lock emptying system together with the flow condition downstream, in the boats waiting zone close to the lock gate (Fig. 2). The choice between numerical or physical modeling approach is justified by the nature of the flow to be represented: where the flows are open channel and when inertia effects are of main importance, numerical modeling has been assessed as reliable. Besides, physical modeling has been chosen to study mixed flow configurations with high influence of turbulence.

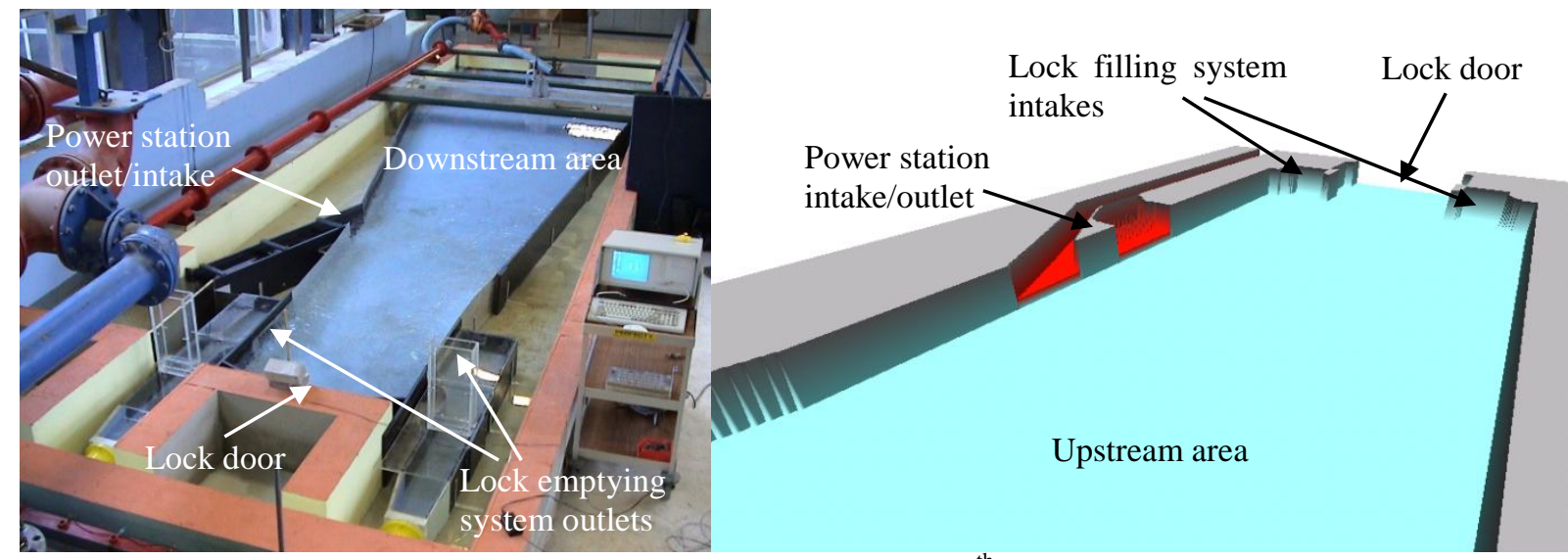

Figure 2: 1:23.33 scale model of the downstream part of the Lanaye $4^{\text {th }}$ lock (left) and numerical model of the upstream part (right).

\subsection{Design of a water intake}

The third example to prove that composite modeling provides a better answer to specific problems than a single approach study concerns the design studies of a water intake. In the case depicted here, most of the hydraulic problems deal with free surface flows in the reservoir and the water intake convergent. Such flows are well handled by numerical modeling tools. These last ones enable also sediment transport and deposition analysis [3]. However, a specific problem arises at the transition between the free surface flow channel and the penstock to the power plant, downstream of the water intake, with possible vortex apparition. This critical issue has also to be addressed by the hydraulic studies of the project. However, numerical models are 
yet under development to model with confidence such flow transition, especially when vortex formation and the associated problem of air entrainment have to be considered. Moreover, it is also difficult to perform reliable scale modeling of such phenomenon if a too small scale factor is used.

In this context, it is obvious that both numerical and physical models are needed to provide the best answer. Numerical modeling has been applied to design the water intake up to the downstream extremity of the convergent, while a 1:14.58 scale model of reduced layout on the real project has been built to address the specific problem of the flow transition and vortex (Fig. 3). Upstream boundary condition of the physical model (velocity field) has been provided by the numerical model. The large scale physical model enables to design safely an efficient anti-vortex system.

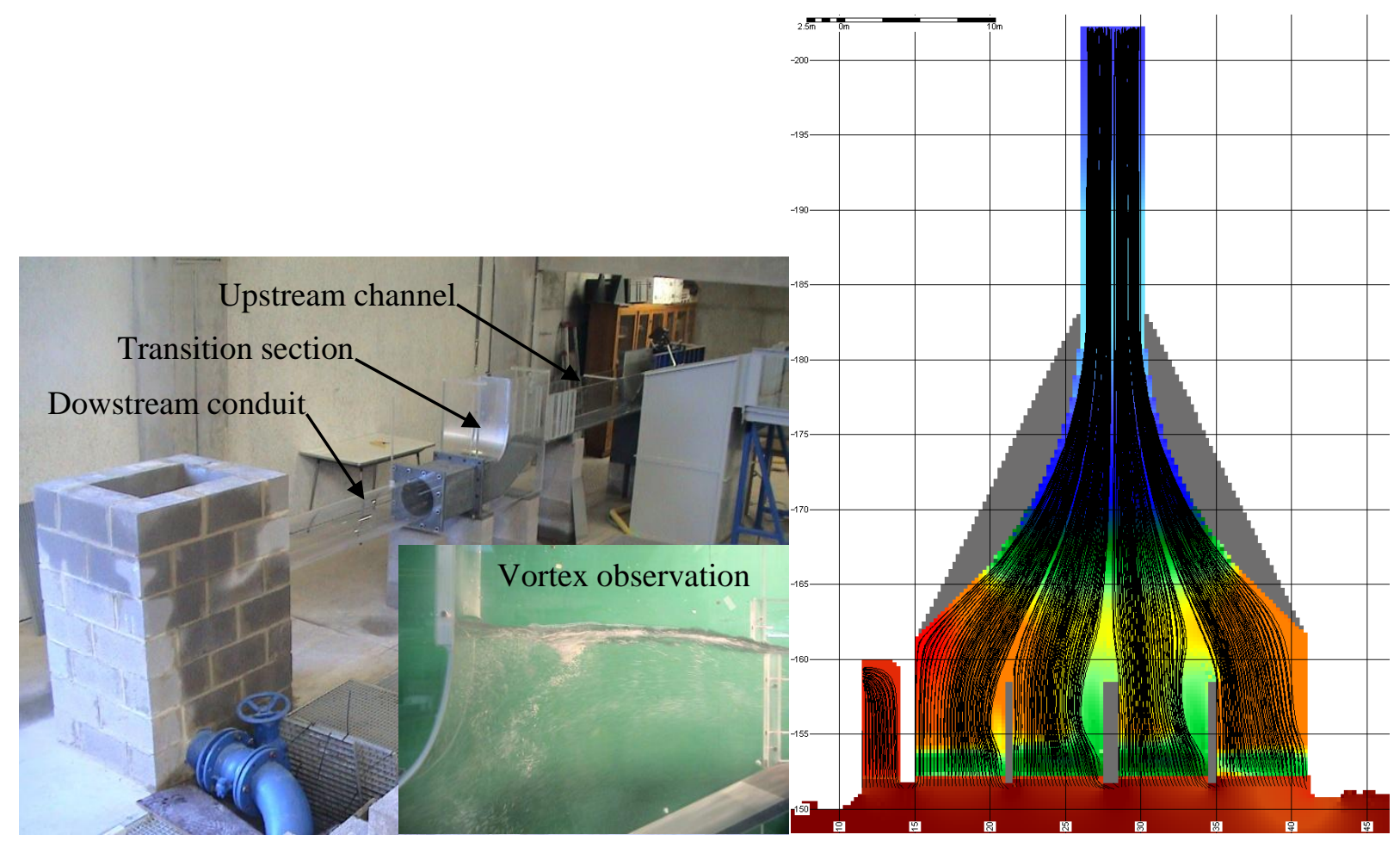

Figure 3: 1:14.58 scale model of the transition section (left) and flow trajectories in the convergent by means of 2D numerical modeling (right).

\section{MAXIMISING THE EFFICIENCY OF EXPERIMENTAL TESTS}

Numerical models are more flexible than physical models and they generally enable more easily results extraction as no measurement device is needed. In that way, it is less time consuming to perform numerical modeling than physical experiments, in particular regarding parametric studies. Such an observation suggests that composite modeling application may be used to maximize the efficiency of experimental tests by reducing the range of variation of the unknown parameters to be tested.

This approach has been successfully applied in the framework of a study about Piano Key Weirs (PKW) with the objective to highlight the most influencing geometric parameters of the structure as well as their best variation interval depending on various criteria related to the weir design [8].

The PKW is a particular shape of labyrinth weir, developed by Lempérière [2], using up- and/or downstream overhangs to limit its basis length and enable its use directly on a concrete dam crest. The PKW proved to be a cost effective solution both for rehabilitation and new dam projects, in which space and available reservoir segment are limited compared to the design discharge to be released. The first scale model studies showed that this new type of weir can be far more efficient than a traditional ogee-crested weir at constant head and crest length on the dam and even more efficient than a labyrinth weir with the same horizontal shape. Although the first prototype size PKWs have been built by "Electricité de France (EDF)" in France since 2006 (see for instance [10]), the definition of the optimal geometry of the structure remains 
poorly described. In this framework, a combined experimental and numerical study, exploiting advantages of the two methods, has been undertaken at the Laboratory of Engineering Hydraulics of the University of Liege to improve the understanding of the flow over this new type of weir and to set up efficient design rules to predict its discharge capacity [9].

In a first step, a 1:10 scale model of a PKW has been exploited in a wide range of discharges to investigate the basic flow features on the structure. The tests provided dense data about the release efficiency of the weir. They also enabled to define the transitions between different flow types on the weir crests (the outlet, lateral and inlet ones) and to characterize the flow on the structure in terms of velocities, pressures, free surface levels and flow patterns [9].

On the basis of the conclusions of the large scale physical tests, it has been possible to develop a 1D model able to rapidly provide a good evaluation of the hydraulic efficiency for a given PKW geometry [4]. From weir geometry, the solver computes the flow over half a unit of the weir for a given range of discharge upstream of the structure. The computation provides the water level upstream of the weir depending on the discharge and the distribution of water depth and discharge along both the inlet and the outlet. Comparison of the numerical results with experimental data for varied geometries and from varied Laboratories (Laboratoire National d'Hydraulique et Environnement - EDF, Chatou, France; Hydraulic Department of Biskra University, Biskra, Algeria and Laboratoire d'Hydraulique des Constructions - ULg, Liege, Belgium) showed a better than $10 \%$ accuracy on weir hydraulic efficiency for a wide range of upstream head (Fig. 4).

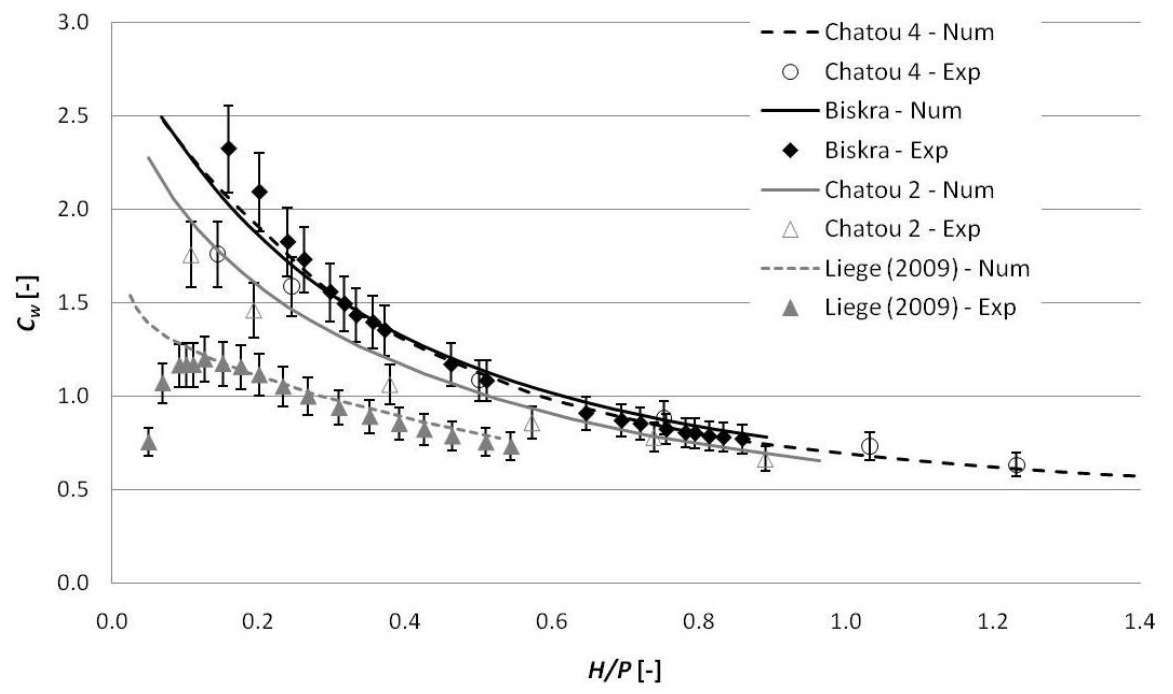

Figure 4: Comparison between experimental and numerical non-dimensional head $H / P$ versus discharge coefficient $C_{w}$ curves for physical models from Chatou (France), Biskra (Algeria) and Liege (Belgium). $\pm 10 \%$ variation bars on the experimental discharge coefficients.

Following the previously depicted large scale model study and the numerical model development, geometric parameters analysis has been performed on the basis of preliminary numerical modeling followed by systematic scale model tests. The conclusions of the large scale model study showed that three nondimensional parameters are particularly important regarding the PKW hydraulic capacity. In order to decrease the time of the parametric physical investigations, numerical modeling has been used to define a pertinent range of variation for each of these parameters. Subsequently, new physical tests have been performed to confirm and optimize the numerical results. In addition to valuable insights into optimal geometric ratios, the parametric tests enable to set up and validate an analytical formulation for the PKW head/discharge relation.

\section{CONCLUSIONS}

Physical modeling and numerical modeling are two efficient analysis approaches in hydraulic engineering. The interactive application of both methods, called composite modeling, is obviously the more 
effective response to most flow problems analyses as it enables to combine the inherent advantages of both approaches, which are complementary.

On the basis of several application examples, it is shown that composite modeling may be useful to increase the scale factor of physical models by reducing the layout of the real structure to modeled, to provide a better answer to specific problems than a single approach study or to maximize the efficiency of experimental tests by reducing the range of variation of the unknown parameters to be tested.

These works prove thus that composite modeling may increase the overall quality and scope of hydraulic analyses while decreasing the delays and possibly the costs. Such an approach should thus be promoted in the field of hydraulics engineering.

\section{REFERENCES}

[1] ASCE (2000). - Hydraulic modeling - Concepts and practice. ASCE Manuals and reports on engineering practice No. 97. Reston, Virginia: ASCE.

[2] Blanc, P. \& Lempérière, F. (2001). Labyrinth spillways have a promising future. International Journal of Hydropower and Dams, 8 (4), 129-131.

[3] Dewals, B.J., Rulot, F., Erpicum, S., Archambeau, P. \& Pirotton, M. (2012). Long-term sediment management for sustainable hydropower. Comprehensive Renewable Energy. Volume 6 - Hydro Power, Elsevier, Oxford.

[4] Erpicum, S., Machiels, O., Archambeau, P., Dewals, B.J. \& Pirotton, M. (2010). 1D numerical approach to model the flow over a Piano Key Weir (PKW), in Proc. of SimHydro 2010: Hydraulic modelling and uncertainty, 2-4 June 2010, Sophia Antipolis, France.

[5] Erpicum, S., Nagel, V. \& Laugier, F. (2011). Piano Key Weir design study at Raviege dam. Labyrinth and Piano Key Weirs - PKW 2011, CRC Press Taylor \& Francis group, 43-50.

[6] Erpicum, S., Archambeau, P., Dewals, B.J. \& Pirotton, M. (2011). Efficient hydraulic numerical modeling with multiblock grids and linked models. La Houille Blanche, 3, 56-62.

[7] Erpicum, S., Dewals, B. J., Vuillot, J.-M., Archambeau, P., \& Pirotton, M. (2012). The Taoussa Project (Mali): an example of effective composite modeling. Proc. of the $4^{\text {th }}$ IAHR Int. Symp. On Hydraulic structures, 9-11 February 2012, Porto, Portugal.

[8] Erpicum, S., Machiels, O., Dewals, B.J., Pirotton, M. \& Archambeau, P. (2012). Numerical and physical hydraulic modelling of Piano Key Weirs. Proc. of the $4^{\text {th }}$ Int. Conf. on Water Resources and Renewable Energy Development in Asia, 26-27 March 2012, Chiang Mai, Thailand.

[9] Machiels, O., Erpicum, S., Dewals, B.J., Archambeau, P. \& Pirotton, M. (2011). Experimental observation of flow characteristics over a Piano Key Weir. Journal of Hydraulic Research, 49(3), 359366.

[10] Vermeulen, J., Laugier, F., Faramond, L. \& Gille, C. (2011). Lessons learnt from design and construction of EDF first Piano Key Weirs. Labyrinth and Piano Key Weirs - PKW 2011, CRC Press Taylor \& Francis group, 215-224. 